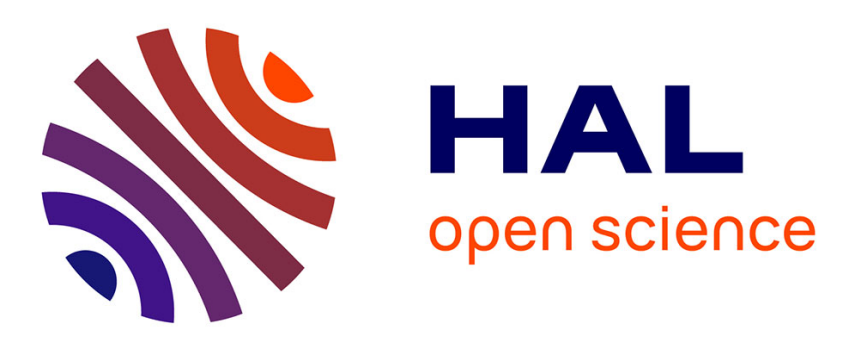

\title{
Analysis of dense coregistration methods applied to optical and SAR time-series for ice flow estimations
}

Laurane Charrier, Pierre Godet, Clément Rambour, Flora Weissgerber, Simon Erdmann, Elise Colin Koeniguer

\section{- To cite this version:}

Laurane Charrier, Pierre Godet, Clément Rambour, Flora Weissgerber, Simon Erdmann, et al.. Analysis of dense coregistration methods applied to optical and SAR time-series for ice flow estimations. 2020 IEEE Radar Conference (RadarConf20), Sep 2020, Florence, Italy. pp.1-6, 10.1109/RadarConf2043947.2020.9266643 . hal-03103824

\section{HAL Id: hal-03103824 \\ https://hal.science/hal-03103824}

Submitted on 8 Jan 2021

HAL is a multi-disciplinary open access archive for the deposit and dissemination of scientific research documents, whether they are published or not. The documents may come from teaching and research institutions in France or abroad, or from public or private research centers.
L'archive ouverte pluridisciplinaire HAL, est destinée au dépôt et à la diffusion de documents scientifiques de niveau recherche, publiés ou non, émanant des établissements d'enseignement et de recherche français ou étrangers, des laboratoires publics ou privés. 


\title{
Analysis of dense coregistration methods applied to optical and SAR time-series for ice flow estimations
}

\author{
Laurane Charrier*†, Pierre Godet*, Clément Rambour* ${ }^{* \ddagger}$, Flora Weissgerber*, Simon Erdmann*, and Elise Colin Koeniguer* \\ * Onera, Université Paris-Saclay † Université Savoie Mont-Blanc, LISTIC $\quad \ddagger$ CNAM, CEDRIC, Paris
}

\begin{abstract}
This article considers the application of two dense coregistration algorithms to the estimation of ice flow. These algorithms estimate displacements at each pixel of the image and can be applied to pairs of radar, optical and radar/optical images. This flexibility combined with the dense estimation should improve both spatial and temporal resolutions of glacier displacement maps. Several tests are carried out on Sentinel-1 and Sentinel-2 images on Totten glacier in Antarctica. We assess the reliability of the considered algorithms by applying them to real and emulated pairs of images based on displacement fields previously estimated in the literature.

Index Terms-SAR images, optical images, coregistration, optical flow, deep learning, dense, multi-modal, ice flow, glaciers
\end{abstract}

\section{INTRODUCTION}

A common critical step in glaciology is the automated computation of velocity maps of glaciers from remote sensing images. Velocity maps are necessary to precisely monitor ice dynamics, to infer sub-glacial processes and/or ocean forcing and to derive other products such as mass-balance or strain rates when the amount of data is sufficient.

Operational methods produce velocity maps with a lower spatial resolution than the spatial resolution of the images [7], [8], [13]. Indeed, most of the existing coregistration methods in glaciology rely on the correlation estimation between two images. Usually, these methods are time-consuming, so the estimation is limited on a sub-grid of pixels. The sampling, i.e., the spatial frequency at which the algorithm estimates the offset tracking, is higher than the pixel size [13] [7]. However, a low resolution can be a limit to analyze spatial discontinuities of ice speed [2] or to derive other products such as strain rates [1]. Here, we propose to analyze results obtained with dense algorithms that estimate a displacement at each pixel. Such displacement is expected to increase the spatial resolution of the velocity time-series.

Besides, operational methods only tackle SAR or optical images [7], [8], [13]. In this paper, we explore multi-modal algorithms that can deal with both optical and SAR images. The complementary between SAR and optical images is expected to increase the temporal resolution of the velocity time-series.

The first considered method is the coregistration algorithm GeFolki [3], an optical flow method that has shown high accuracy, high resolution, and high robustness on urban [15] and forest [4] remote sensing images. Optical flow methods have already shown relevance and limits to compute glacier velocity on optical images [1], [18]. Moreover, a study on Antarctica images demonstrated the ability of GeFolki to deal with glaciers SAR images [6]. Therefore, GeFolki appears to be an interesting method to explore.

The second considered method, more recent, is a deeplearning-based dense coregistration algorithm dedicated to remote sensing images [17]. Trained on SAR and optical images obtained both with airborne and spaceborne sensors, this algorithm called PWC-Net-multimodal shown better results than GeFolki on SAR, optical, and SAR/optical image coregistration on continental areas. PWC-Net-multimodal has been trained with synthetic displacement fields computed from a digital elevation model, to correct offsets due to relief. Therefore, this algorithm is initially not intended to operate on terrain or glacier movements. However, to re-train this type of algorithm on glacier flows would be a lengthy process. The generation of realistic synthetic glacier flows would require a full study that is beyond the scope of this paper. Therefore, in the first place, we propose to test this network on glaciers without another training step.

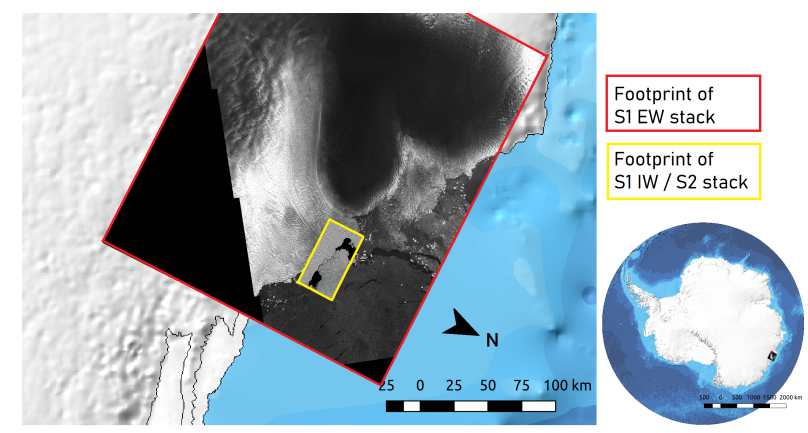

Fig. 1. Totten Glacier footprints. The red footprint corresponds to the stack of Sentinel-1 EW images and the yellow footprint to the stack of Sentinel-1 IW and Sentinel-2 images.

This study aims to carry out the analysis of the two different methods, in the area of the Totten glacier, a stable and major outlet, where GeFolki has already shown convincing results on radar images [6]. We consider dense coregistration on optical and radar images, to assess the potential of the different methods available in terms of producing velocity series estimates. Several criteria are evaluated: 
- the ability to produce estimates using SAR/SAR pairs; optical/optical, or SAR/optical.

- the accuracy on the estimated velocity

- the spatial resolution of the velocity fields

- the robustness of the estimates.

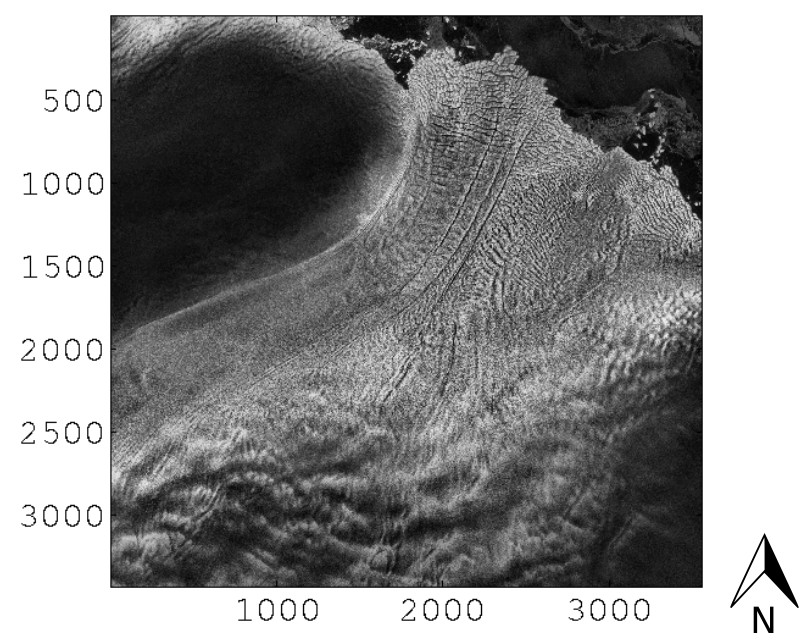

Fig. 2. Sentinel-1 EW (40m) image from the 3 November 2017 on Totten Glacier. The level of the product is the Levell GRD.

\section{DATASET AND PREPROCESSING}

We have created different Sentinel-1 and Sentinel-2 datasets on Totten glacier $\left(67^{\circ} \mathrm{S} 116^{\circ} \mathrm{E}\right)$ in Antarctica. Totten glacier is one of the major contributor of mass loss in East-Antarctica. It drains a sector $537.900 \mathrm{~km}^{2}$ in size, mostly grounded below sea level. [8], [10] reveal that Totten glacier increased in velocity between 2001 and 2007 but remained stable since, likely due to a prograde bed [14]. This stability allows us to validate our measured velocities with previous works. Because the methods have never been applied to glaciers, Totten glacier would be a relevant first case to study.

The creation of a dataset was subject to various constraints related to the footprints and dates of acquisitions. Figure 1 shows the areas related to the two footprints used. The Sentinel-1 EW (about $40 \mathrm{~m}$ per pixel) data have large footprints that allow us to see the entire glacier in motion. The Sentinel-1 IW and Sentinel-2 data are better resolved (about $10 \mathrm{~m}$ per pixel), but the footprints do not cover such a large area of the glacier. Furthermore, the dates of acquisitions are not necessarily overlapping. Sentinel-1 EW images are mainly available for the year 2017 whereas Sentinel-2 images without clouds were mainly acquired in 2018. As for Sentinel-1 IW images, it covers the years 2017 and 2018. Taking all these constraints into account, we created two sets of data:

- The first set is composed of a dozen of Sentinel-1 images covering a large area, in EW mode, for the year 2017 (cf. Figure 2).

- The second set is composed of Sentinel-1 images, which cover a much more restricted area, in IW mode, over 18 months from June 2017 to the end of 2018, and 4 Sentinel-2 images acquired in 2018 ( $c f$. Figure 7).
Images were downloaded using the platform Google Earth Engine, in Level 1 GRD for radar images and Level 1C for optical images. The Terrain Correction step offered by SNAP is already applied to the images.

On the first stack, we make a comparison of the two dense co-registration algorithms GeFolki and PWC-Net-multimodal, while on the second stack, we investigate mainly GeFolki and its capacity to handle optical images.

A colored composition was produced using the REACTIV algorithm [5] to quickly visualize the second stack of IW images. Figure 3 allows us to view all the images in the stack. A pixel where the signal is not stable in all the images trough the time is saturated. Each color is representative of one specific date.

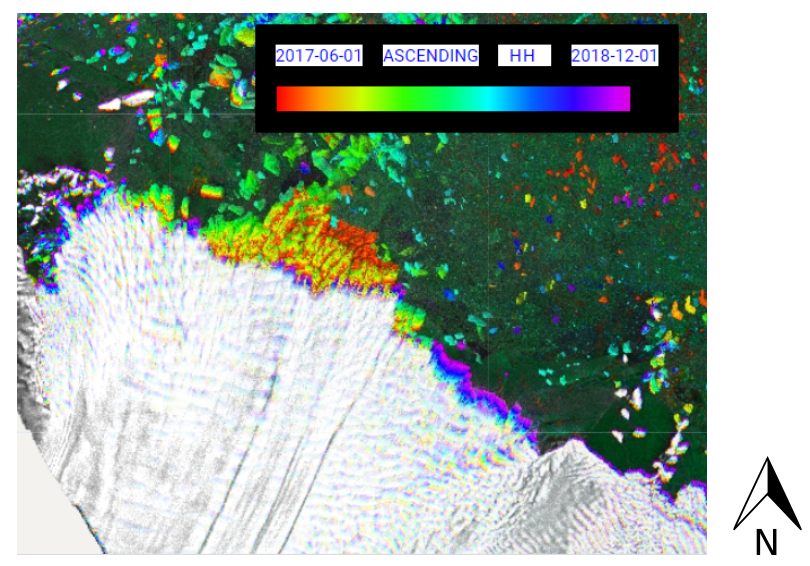

Fig. 3. Colored composition of the Sentinel-1 IW images using REACTIV algorithm [5]

\section{METHODS}

In this section, we briefly present the two evaluated algorithms to understand their respective advantages and drawbacks for glacier displacements estimation.

The first considered method, GeFolki, is an optical flow algorithm that has already shown accuracy as good as correlation methods [4]. The second considered algorithm, PWC-Netmultimodal, is a more modern $\mathrm{CNN}$ method. Both are dense and multi-modal coregistration methods.

\section{A. GeFolki}

GeFolki is a dense flow estimation algorithm based on optical flow equations. Its principles are widely described in [4], [15], but we emphasize here some of them.

GeFolki is based on the Lucas-Kanade paradigm [11]. It belongs to local or window-based approaches of optical flow: the flow estimated in one pixel is defined as the minimizer of a criterion computed over a local window centered on this pixel. Minimization is done by an iterative Gauss-Newton strategy based on first-order Taylor expansion of the image intensity around a previous guess of optical flow. The first-order Taylor expansion assumes a constant flow over the local window used, therefore spatial discontinuities inside this window are likely to be smoothed. GeFolki is also multi-resolution, i.e., uses 
a pyramid of images to compute the optical flow at varying scales following a coarse-to-fine strategy. The minimization at each level of resolution, called the level of the pyramid, is initialized with the flow from the previous level. Moreover, at each pyramid level, the window's radius can also iteratively vary. Finally, a Rank transform is applied to the images before minimization. Each pixel is replaced by the number of neighboring pixels with an intensity lower than its intensity. This specificity is of a broad interest in glaciers applications. It allows us to be more robust to radiometric changes. Indeed, the radiometric changes often cause failure in glaciers applications: [1], [18] show that, on optical images, irradiance variations between autumn and spring at high latitudes or reflection intensity changes due to different snow "ages" were major sources of failure for optical flow methods.

In practical terms, GeFolki has several parameters to tune: the number of pyramid levels, the radius of the windows, the size of the rank filter, and the number of iterations. The number of pyramid levels should be set according to the expected maximum of flow magnitude. Regarding the radius, the higher it is, the more robust is the algorithm, but the smoother is the result. When increasing the radius, the precision increases, but the resolution, i.e. the spatial frequency at which the precision can be maintained, decreases. We will discuss later a strategy to adjust precisely GeFolki parameters according to each type of image.

In previous studies, GeFolki has been tested on highresolution radar/radar or radar/optical images of urban areas [15] and optical/radar images of vegetated areas [3]. It has proven to be fast in comparison to other image correlation strategies. [4] obtain with GeFolki a flow for a 5,000 x 5,000 pixel image on a Intel(R) Core(TM) i7-2620M @ 2.80GHz with a GPU implementation in hundreds of milleseconds.

\section{B. PWC-Net-multimodal}

The PWC-Net-multimodal algorithm is based on the PWCNet architecture, a state-of-the-art Convolutional Neural Network (CNN) for optical flow estimation in computer vision [16].

The original PWC-Net uses a siamese encoder for the two input images. On the opposite, PWC-Net-multimodal has two different encoders: one specialized for optical images, the other for radar images. It is then able to deal with heterogeneous image pairs. PWC-Net-multimodal is trained on a remote sensing image dataset composed of radar and optical images from different resolutions and frequencies (low-resolution Sentinel-1 and Sentinel-2 images, and on higher-resolution aerial images from American UAVSAR and NAIP programs), covering continental areas. The training step mixes the coregistration of optical/optical, radar/radar, and radar/optical images. The generalization ability of the algorithm has proved to be very good on $\mathrm{X}$ band images with higher spatial resolution. However, the network has been trained with synthetic flow indexed on the SRTM DEM. The goal was to fix errors in the coregistration between images, assumed to be caused by errors in projection over the topography. Therefore, it has never been
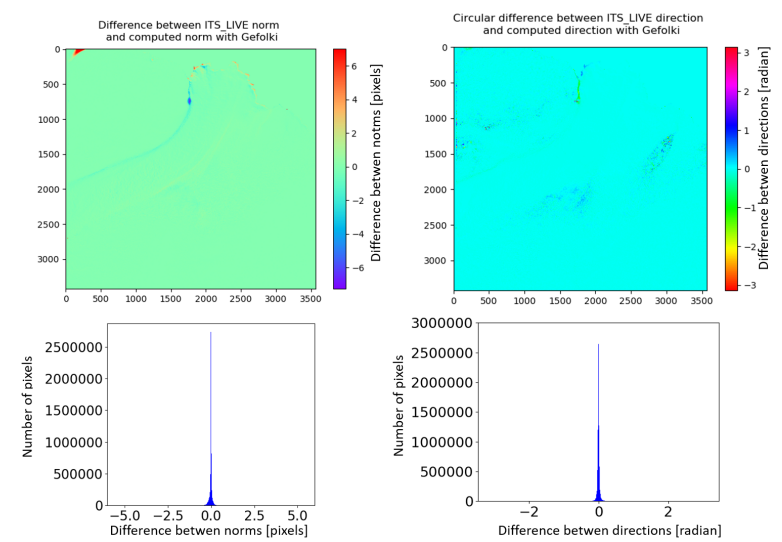

Fig. 4. Difference between the flow from ITS_LIVE and the flow computed by GeFolki on an emulated pair of Sentinel-1 EW images where the master image is from the 2 May 2017. GeFolki parameters are radius $=[64,32,16,8]$, levels $=4$, rank $=4$, iteration $=2$. The difference in magnitude is displayed on the left and the circular difference in direction on the right. Histograms are displayed below the corresponding difference.

trained neither on ice-sheets and glaciers nor when the flow is decorrelated from the relief.

\section{ANALYSIS}

\section{A. Comparison of GeFolki and PWC-Net-multimodal on Sentinel-1 EW images}

First, we study the performances of GeFolki and PWCNet-multimodal in controlled conditions. We try to find an emulated displacement to assess the ability of both algorithms to retrieve a known flow. Indeed no ground truth measurements are available near Totten glacier. Therefore, we apply a flow from the literature to a Sentinel-1 EW master image to obtain an emulated slave image. Here, we use a master image from the 2 May 2017 and a time-average velocity map called ITS_LIVE published by [8] with a resolution of $120 \mathrm{~m}$ (roughly 3 pixels on Sentinel-1 EW images). The covering area is represented in Figure 9. The velocity map is reprojected and resampled, and we convert its values to obtain a displacement expressed in pixels in the geometry of the master image, for a period equivalent to 6 months.

On the one hand, performances of GeFolki depend on the chosen parameters, especially the radius and number of pyramid levels. Therefore, we pick the parameters which minimize the criterion MSE (Mean Square Error) and MAE (Mean Absolute Error) between the reference flow and the resulted flow. We obtain the highest MSE and MEA when we set the number of pyramid $\mathrm{L}$ such as $2^{L}<D$, where $D$ is the maximum of expected flow magnitude. For Sentinel-1 EW images, the best parameters appear to be: radius $=[64,32,16,8]$, levels $=4$, rank=4, iteration=2. It allows us to reach a MAE of $610^{-2}$ pixels and MSE of $910^{-2}$ pixels for the flow magnitude. To quantify errors in angles which are circular variables varying modulo $2 \pi$, the use of circular statistics is more suitable. Thus the mean of the circular difference between flow directions is $610^{-4} \mathrm{rad}$. This gives an idea of 
the accuracy of GeFolki. The results show that GeFolki can retrieve an emulated flow in most parts of the image ( $c f$. Figure 4). The most difficult areas correspond to the border of the ice stream and the ice front where a break in velocity occurs. This is likely because GeFolki is locally constrained. On the other hand, PWC-Net-multimodal seems to retrieve the known flow with less accuracy, in particular in the Dome area and near the ice front ( $c f$. Figure 5). The MAE is of 1.49 pixels and MSE of 10.41 pixels for the flow magnitude. The mean of the circular difference between flow directions is of $2.610^{-2} \mathrm{rad}$.

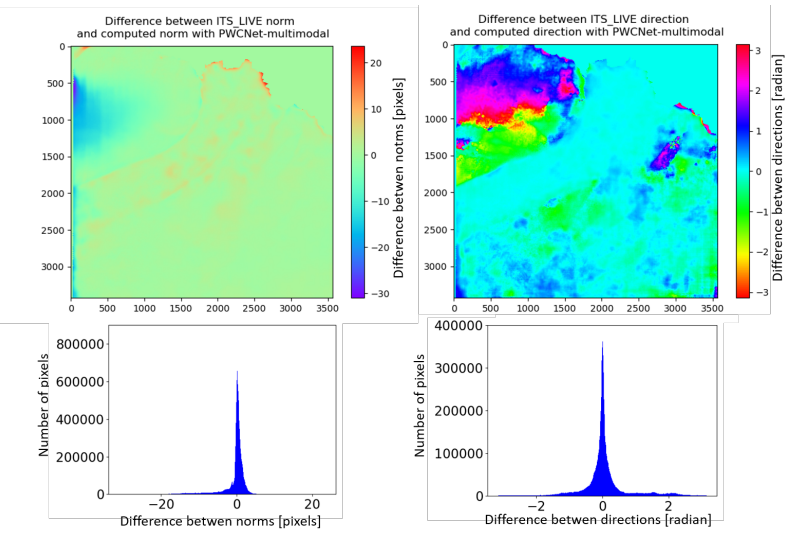

Fig. 5. Difference between the ITS_LIVE flow and the flow computed using PWC-Net-multimodal on an emulated pair of images. The master image is from the 2 May 2017. The difference in magnitude is displayed on the left and the circular difference in direction on the right. Histograms are displayed below the corresponding difference.

Then, we measure a real displacement between two Sentinel-1 EW images from the 2 May 2017 and the 3 November 2017 (pair 1) using the previous GeFolki parameters. The results are shown in Figure 6. According to [10], we expect the flow magnitude to be around $1800 \mathrm{~m} / \mathrm{yr}$ near the ice front and $800 \mathrm{~m} / \mathrm{yr}$ in the ice stream. We convert the estimated displacement in pixel to a displacement in $\mathrm{m} / \mathrm{yr}$. For this analysis, we assume that geometric distortion and errors due to ortho-projection are negligible. We find a flow magnitude from 1600 to $2000 \mathrm{~m} / \mathrm{yr}$ near the ice front, and from 500 to 1000 $\mathrm{m} / \mathrm{yr}$ in the ice stream. The direction, which is South-West / North-East in most of the image, is also a consistent result: the ice stream flows toward the ocean. However, we notice that the direction found in the Western Southern part of the image is the opposite of the expected one. We try to compute a displacement on other pairs: 1 July 2017 - 3 November 2017, 1 July 2017 - 4 August 2017 and 2 May 2017 - 4 August 2017 and find the same behavior. This point needs further investigation.

TABLE I

GeFolKi PARAMETERS USED IN THIS STUdy FOR THE SENTINEL-1 EW, SENTINEL-1 IW AND SENTINEL-2 IMAGES

\begin{tabular}{|ccccc|}
\hline Type of images & Radius & Level & Rank & Iteration \\
\hline Sentinel-1 EW & {$[64,32,16,8]$} & 4 & 4 & 2 \\
Sentinel-1 IW & {$[128,64,32,16,8]$} & 7 & 4 & 2 \\
Sentinel-2 & {$[32,16]$} & 4 & 4 & 8 \\
\hline
\end{tabular}

Furthermore, several criteria can be used to evaluate an estimated displacement. Here, we propose to have a look at the back-forward flow, also called residual. This is the difference between the computed displacement from the image 1 to 2 and its opposite, the displacement from image 2 to 1 . For the pair 1 , the residual of the $\mathrm{x}$-component of the flow has a median value of 0.14 pixels and a standard deviation of 0.35 pixels. Regarding the y-component of the flow, the median value of the residual is 0.14 pixels, and its standard deviation is 0.25 pixels. By doing the same process for all the four evaluated pairs of images, we find, on average, a residual with a median value of 0.14 pixels for both the $\mathrm{x}$-component and the $\mathrm{y}$ component. That gives an idea of the precision of GeFolki for these pairs of images. This criterion can also help to reject the outliers, i.e. pixels where the residue is above a given threshold.

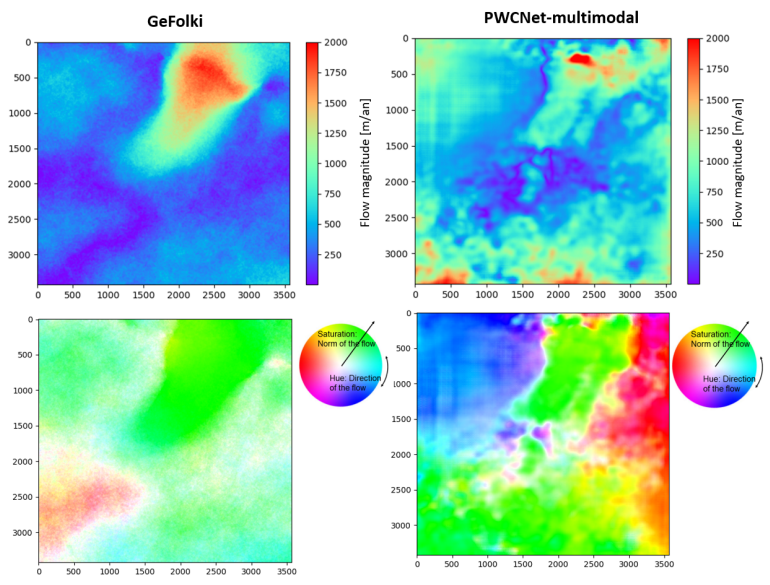

Fig. 6. Flow computation on a real pair of Sentinel-1 images. The master image is from the 2 May 2017 and the slave image from the 3 November 2017. Results of GeFolki (left), and of PWC-Net-multimodal (right).

Finally, we compare the performances of GeFolki and PWC-Net-multimodal on the same pair of real images $(c f$. Figure 6). PWC-Net-multimodal finds a high displacement (between 1900 and $2300 \mathrm{~m} / \mathrm{yr}$ ) near the ice front, which is quite consistent with GeFolki's results. However, this high displacement seems to be too concentrated to be realistic. We also notice a huge displacement at the bottom of the image. Since this latest result doesn't have any glaciology meaning, it could be due to an over-learning of relief elements by the algorithm.

To conclude, GeFolki has proven its ability to retrieve a given glacier flow. Even if the reference flow used has a resolution of $120 \mathrm{~m}$ and corresponds to a time-average, we can assume that it remains closed to a real glacier displacement. The emulation of such displacement could, therefore, be a way to choose GeFolki parameters. These parameters need to be changed depending on the type of image (optical or SAR) and its resolution. The application of GeFolki on a real pair of images leads to a coherent flow magnitude in the whole image and a coherent direction on most of the ice-sheet. 
The results give a first idea of the precision and accuracy of the algorithm for ice flow estimations. However, PWC-Netmultimodal does not seem to be suitable to compute a glacier displacement without another dedicated training. It is likely because the training dataset uses a SRTM digital elevation model to emulate the deformation. This choice was relevant to align two images with no natural source of displacement, but need to be adapted with a glacier flow database for glaciology purpose. In this case, we think that PWC-Net-multimodal could even be more robust than GeFolki, as it was already the case in our previous experiments [17]. Both algorithms still appear to be high-speed methods to retrieve a flow and allow a dense coregistration.

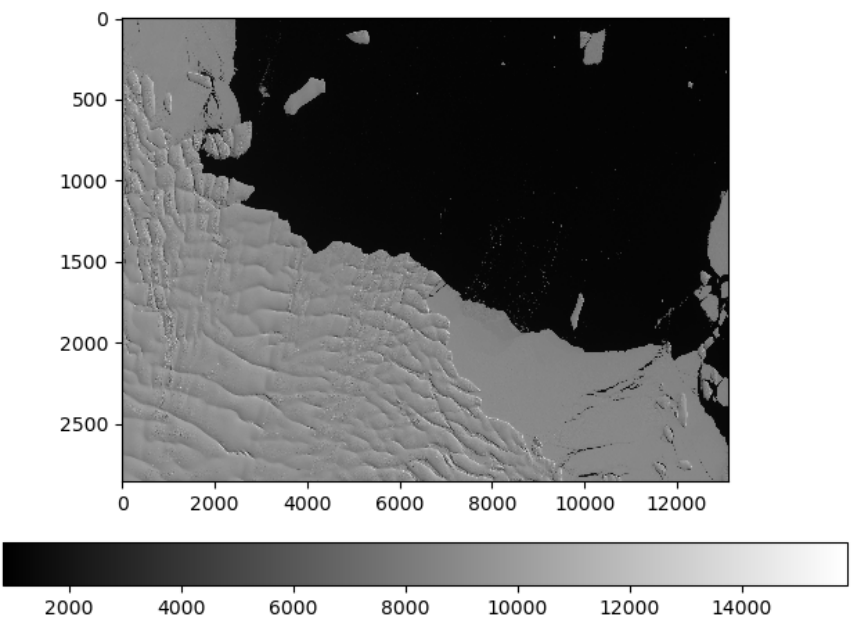

Fig. 7. Sentinel 2 image $(10 \mathrm{~m})$ from the 31 January 2018 on Totten Glacier The level of the product is the Level 1C. The values correspond to Top Of the Atmosphere (TOA) reflectance.

\section{B. Comparaison of GeFolki on optical and SAR images}

The GeFolki algorithm can handle heterogeneous images. Therefore, we compare the results of GeFolki on Sentinel-2, Sentinel-1 IW, and Sentinel-1 EW images for the same period. The studied footprint appears in yellow in Figure 1. One of the corresponding Sentinel-2 images is shown in Figure 7.

For Sentinel-2, we use the blue channel (10 $\mathrm{m}$ of spatial resolution and 12 bit of radiometric resolution). For Sentinel$1 \mathrm{EW}$ and IW, the channel corresponds to the polarimetry $\mathrm{HH}$. We choose GeFolki parameters using the methodology previously presented in section A. For Sentinel-1 IW, the spatial resolution is higher than the one of Sentinel-1 EW, therefore we should increase the levels of pyramids ( $c f$. Table I). For Sentinel-2, the pair of images corresponds only to a small baseline, so we pick the parameters listed in Table I.

Results on Sentinel-1 IW are similar to results on Sentinel1 EW: we measure roughly the same flow magnitudes and directions in the same locations ( $c f$. Figure 8). Since the resolution of the Sentinel-1 IW image is higher, we notice more details on the resulted velocity map. Results on Sentinel2 and Sentinel-1 IW are also very correlated on the ice-sheet parts (Bottom Left part of the image) ( $c f$. Figure 9).

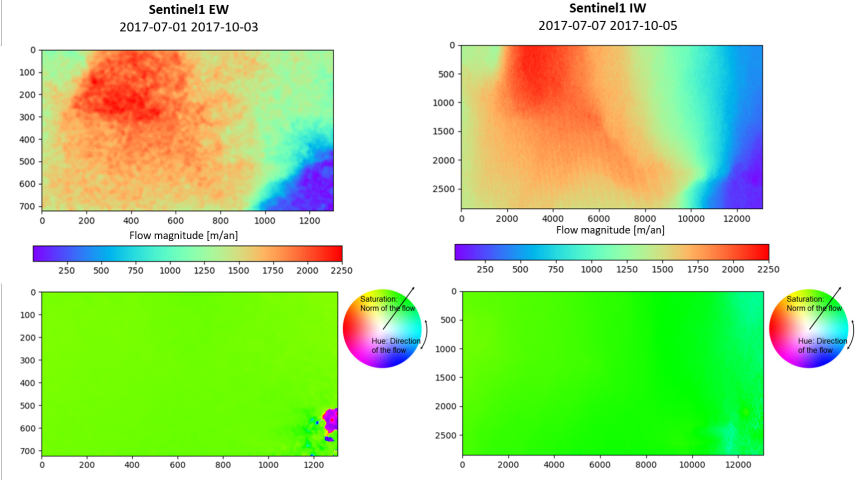

Fig. 8. Comparison between a flow estimation with GeFolki on real Sentinel1 EW images (left) and Sentinel-1 IW images (right) covering roughly the same period. The GeFolki parameters used are listed in Table I. For Sentinel-1 IW, the master image is from the 7 July 2017 and the slave image from the 5 October 2017. For Sentinel-1 EW, the slave image is from the 3 July 2017 and the slave image from the 3 October 2017

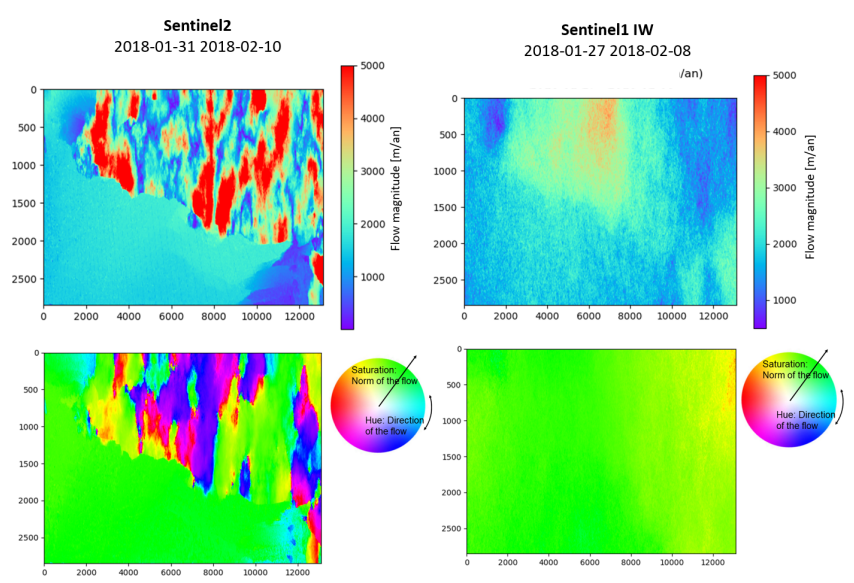

Fig. 9. Comparison between a flow estimation with GeFolki on real Sentinel2 images (left) and Sentinel-1 IW images (right) overing roughly the same period of time. The GeFolki parameters used are listed in Table I. For Sentinel$1 \mathrm{IW}$, the master image is from the 27 January 2018 and the slave image from the 8 February 2018. For Sentinel-2, the slave image is from the 31 January 2018 and the slave image from the 10 February 2018.

We have also tried to coregistrate a pair of radar/optical images. The results are promising but still need to be improved. Indeed, the flow direction is consistent but the flow magnitude is not. This is probably due to a difference in the acquisition geometry. When considering qualitatively the superposition of close images, it appears that the shadows are not projected in the same way. Therefore, GeFolki tries to compensate for the misalignment of the shadow projections instead of a real feature.

\section{CONCLUSiON}

This paper aimed to investigate the capabilities of current coregistration methods designed to handle various images and produce dense displacements for the task of glacier 
displacements estimation. The goal is to explore the possibility of increasing both the spatial and temporal resolution of velocity maps. We have chosen and evaluated two algorithms: GeFolki and PWC-Net-multimodal. GeFolki has already been successfully applied to several other cases, while PWC-Netmultimodal is a more recent algorithm that gave remarkable results on specific cases but still requires further evaluations.

This study confirmed the ability of GeFolki to retrieve an ice flow using optical and SAR images. Five main advantages appear to us compared to other classical coregistration methods such as image matching methods. First, GeFolki already proved to be a fast method in comparison with other correlation methods [4]. It may quickly provide a displacement at each pixel without, for example, the need for previous sparse research as developed in [8]. Secondly, the flow computation relies on several window sizes, for the same pixel. It offers the possibility to take into account different levels of details and to be more robust. Thirdly, GeFolki can directly retrieve a subpixel offset, contrary to cross-correlation methods which need to oversample the images and/or the correlation functions. For instance, the method proposed in [12] uses an oversampling of the image in the Fourier domain through Shanon interpolation. In [9], the authors propose to oversample the correlation function by a factor of 10 . Then, to deal with contrast variations, GeFolki includes a rank filter processing at each level of the pyramid, instead of previously filtering the image with a lowpass filter [7] or a Wallis operator [8]. Finally, GeFolki can even be applied without interferometric conditions. Indeed, it is not sensitive to loss of coherence which is common on SAR images of glaciers and ice-sheets.

PWC-Net-multimodal is a much more recent algorithm than GeFolki. Even if it has never been trained on real displacement cases, this $\mathrm{CNN}$ can retrieve an ice flow that is consistent with GeFolki results in areas where big displacements occur, for instance on the ice fronts. However, several artifacts are spotted in areas of weak displacements outside of the ice stream. Therefore, the results still have to be improved. For that, a good solution could be to design a new training process of CNN. As PWC-Net-multimodal has never been trained on glaciers or ice-sheets, it appears to us that this algorithm could provide very promising results with a dedicated training dataset. A strategy could be to use glacier flows estimated by other algorithms. However, the training step would be impacted by the accuracy and resolution of these velocity maps. Besides, data augmentation techniques could improve performances at a lower cost.

To go further, it would be interesting to apply GeFolki and PWC-Net-multimodal to glaciers with more complexity such as mountain glaciers like alpine glaciers. Furthermore, GPS data from GLACIOCLIM observatory are available in the Alps (https://glacioclim.osug.fr/). It would allow us to give another validation and a measure of the accuracy of our glacier surface velocities. Last but not least, it will be very relevant to compare the performance of GeFolki to other algorithms such us auto_RIFT [ [8]], Pycorr [7] or the modified version of ampcor used by [13].

\section{REFERENCES}

[1] Bas Altena and Andreas Kääb. Weekly glacier flow estimation from dense satellite time series using adapted optical flow technology. Frontiers in Earth Science, 5:53, 2017.

[2] William H Armstrong, Robert S Anderson, and Mark A Fahnestock. Spatial patterns of summer speedup on south central alaska glaciers. Geophysical Research Letters, 44(18):9379-9388, 2017.

[3] Guillaume Brigot, Elise Colin-Koeniguer, Aurélien Plyer, and Fabrice Janez. Adaptation and evaluation of an optical flow method applied to coregistration of forest remote sensing images. IEEE Journal of Selected Topics in Applied Earth Observations and Remote Sensing, 9(7):29232939, 2016.

[4] Guillaume Brigot, Elise Colin-Koeniguer, Aurélien Plyer, and Fabrice Janez. Adaptation and evaluation of an optical flow method applied to coregistration of forest remote sensing images. IEEE Journal of Selected Topics in Applied Earth Observations and Remote Sensing, 9(7):29232939, 2016.

[5] Elise Colin-Koeniguer, Alexandre Boulch, Pauline Trouve-Peloux, and Fabrice Janez. Colored visualization of multitemporal SAR data for change detection: issues and methods. In EUSAR 2018; 12th European Conference on Synthetic Aperture Radar, pages 1-4. VDE, 2018.

[6] Simon Erdmann. Glaciers velocity maps generation through optical flow method. Master's thesis, Ecole des Mines de Paris, Paris, France, september 2018.

[7] Mark Fahnestock, Ted Scambos, Twila Moon, Alex Gardner, Terry Haran, and Marin Klinger. Rapid large-area mapping of ice flow using landsat 8. Remote Sensing of Environment, 185:84-94, 2016.

[8] Alex S Gardner, Geir Moholdt, Ted Scambos, Mark Fahnstock, Stefan Ligtenberg, Michiel Van Den Broeke, and Johan Nilsson. Increased west antarctic and unchanged east antarctic ice discharge over the last 7 years. Cryosphere, 12(2):521-547, 2018.

[9] Ian Joughin. Ice-sheet velocity mapping: a combined interferometric and speckle-tracking approach. Annals of Glaciology, 34:195-201, 2002.

[10] Xin Li, Eric Rignot, Jeremie Mouginot, and Bernd Scheuchl. Ice flow dynamics and mass loss of totten glacier, east antarctica, from 1989 to 2015. Geophysical Research Letters, 43(12):6366-6373, 2016.

[11] Bruce D. Lucas and Takeo Kanade. An iterative image registration technique with an application to stereo vision. In Proceedings of the 7th International Joint Conference on Artificial Intelligence - Volume 2, IJCAI'81, page 674-679, San Francisco, CA, USA, 1981. Morgan Kaufmann Publishers Inc.

[12] Remi Michel and Eric Rignot. Flow of glaciar moreno, argentina, from repeat-pass shuttle imaging radar images: comparison of the phase correlation method with radar interferometry. Journal of Glaciology, 45(149):93-100, 1999.

[13] Romain Millan, Jérémie Mouginot, Antoine Rabatel, Seongsu Jeong, Diego Cusicanqui, Anna Derkacheva, and Mondher Chekki. Mapping surface flow velocity of glaciers at regional scale using a multiple sensors approach. Remote Sensing, 11(21):2498, 2019.

[14] Mathieu Morlighem, Eric Rignot, Tobias Binder, Donald Blankenship, Reinhard Drews, Graeme Eagles, Olaf Eisen, Fausto Ferraccioli, René Forsberg, Peter Fretwell, et al. Deep glacial troughs and stabilizing ridges unveiled beneath the margins of the antarctic ice sheet. Nature Geoscience, 13(2):132-137, 2020.

[15] Aurélien Plyer, Elise Colin-Koeniguer, and Flora Weissgerber. A new coregistration algorithm for recent applications on urban sar images. IEEE Geoscience and Remote Sensing Letters, 12(11):2198-2202, 2015.

[16] Deqing Sun, Xiaodong Yang, Ming-Yu Liu, and Jan Kautz. PWC-Net: CNNs for optical flow using pyramid, warping, and cost volume. In Proceedings of the IEEE Conference on Computer Vision and Pattern Recognition, pages 8934-8943, 2018.

[17] Benjamin Le Teurnier. A coregistration tool for optical and sar images by deep learning methods. Master's thesis, Institut d'Optique Graduate School, Palaiseau, France, september 2019.

[18] Christoph Vogel, Andreas Bauder, and Konrad Schindler. Optical flow for glacier motion estimation. In Proceedings of the 22nd ISPRS Congress, Melbourne, Australia, volume 25, 2012. 